\title{
The neutral gas in the environs of the Wolf Rayet stars in the Circinus OB1 association
}

\author{
E. Giacani and G. Dubner \\ Instituto de Astronomía y Física del Espacio (IAFE), CC 67, Suc. 28, 1428 Buenos Aires, Argentina \\ e-mail: gdubner@iafe.uba.ar
}

Received 17 July 2003 / Accepted 18 September 2003

\begin{abstract}
We have investigated the neutral hydrogen in the direction of the Circinus OB1 association with particular emphasis in the study of the vicinity of the WR stars WR 65 and WR 67. The HI line data were obtained with the Australia Telescope Compact Array with a synthesized beam of $4.0 \times 2$.'7 and a velocity resolution of $1.1 \mathrm{~km} \mathrm{~s}^{-1}$. These data led to the discovery of a large cavity surrounded by an almost complete shell in agreement with the locations and distances of the two WR stars. We propose that the $\mathrm{HI}$ features constitute an interstellar bubble created by the powerful winds of the WR stars and their progenitors. By assuming a distance of $3 \mathrm{kpc}$, we calculate for this $\mathrm{HI}$ bubble a linear radius of $22 \mathrm{pc}$, a swept-up mass of $1600 M_{\odot}$ and an expansion velocity of more than $7 \mathrm{~km} \mathrm{~s}^{-1}$. High angular resolution IRAS infrared data (HIRES) reveal the presence of a ring with good spatial correlation with the HI shell. Eight IRAS protostellar candidates located around the HI cavity suggest that star formation may be taking place in the shell. Further investigation is required to confirm this proposition.
\end{abstract}

Key words. ISM: general - stars: individual: WR 65 - stars: individual: WR 67 - stars: Wolf-Rayet

\section{Introduction}

Wolf-Rayet (WR) are hot, luminous stars that have evolved from massive progenitors of type $\mathrm{O}$. Their stellar winds are one of the most powerful sources of matter and energy of the interstellar medium (ISM). During its WR phase only, the star can release through the stellar wind an energy of up to $10^{51} \mathrm{ergs}$, comparable to the energy injected in a supernova explosion. These winds leave clear signs of their interaction with the ISM shaping the environment dramatically and creating interstellar wind-blown bubbles (a region highly evacuated often surrounded by an outer expanding shell).

These bubbles have been observed as voids in the HI emission distribution in the vicinity of several WR stars (see for example Arnal et al. 1999; Cazzolato \& Pineault 2000; Cichowolski et al. 2001). Generally, these cavities are elongated with the star off-center (Arnal 1992; 2001) and often are surrounded by an HI shell (see for example Cappa et al. 1996; Gervais \& St-Louis 1999). Based on their slow expansion velocity $\left(\sim 10 \mathrm{~km} \mathrm{~s}^{-1}\right)$, the estimated dynamical age of the bubbles generally exceeds the duration of the WR phase, $\left(\leq 5 \times 10^{5}\right.$ years, Schaller et al. 1992), implying that the progenitor $\mathrm{O}$ star plays a major role in the formation of these HI cavities.

In this paper we analize the neutral gas distribution in the local environs of the WR stars Wra 1297 and LSS 3329, that

Send offprint requests to: E. Giacani, e-mail: egiacani@iafe.uba.ar is WR 65 and WR 67, respectively, according to the catalog of van der Hucht (2001). The HI study has been performed with the aim of investigating the action of their strong stellar winds on the surrounding. The study is based on observations carried out with the Australia Telescope Compact Array (ATCA) in a $2.4 \times 2^{\circ} .4$ field centered around $15^{\mathrm{h}} 15^{\mathrm{m}},-59^{\circ}(\mathrm{J} 2000)$, as a part of a survey of the neutral hydrogen in the direction of the bilateral supernova remnant (SNR) G320.4-1.2. This is a region rich in early-type stars and the stars WR 65 and WR 67 appear in the plane of the sky in the same field that the SNR but are about $1.5 \mathrm{kpc}$ closer to the Sun. The results of the study of the distribution and kinematics of the neutral gas associated with G320.4-1.2 have already been presented (Dubner et al. 2002).

WR 67, classified as a WN6 (van der Hucht 2001), is a member of the young open cluster Pismis 20 (Vázquez et al. 1995; Turner 1996; Massey et al. 2001), which is probably related to the Circinus OB1 association (Lortet et al. 1987). WR 65, classified as a WC9 (van der Hucht 2001) is a probable member of the Circinus OB1 association (Lortet et al. 1987). Two more WR stars, namely, WR 66 and WR 68 are also proposed as members of the Cir OB1 association (Lortet et al. 1987), but they lie outside our observed field and will not be studied here. Based on their correlation with the open cluster and the stellar association, the same photometric distance of $3.27 \pm 0.3 \mathrm{kpc}$ is assigned to WR 65 and WR 67 (van der Hucht 2001).

A diffuse ring nebula of $\mathrm{H} \alpha$ emission associated with WR 65 was detected by Marston et al. (1994), although its 


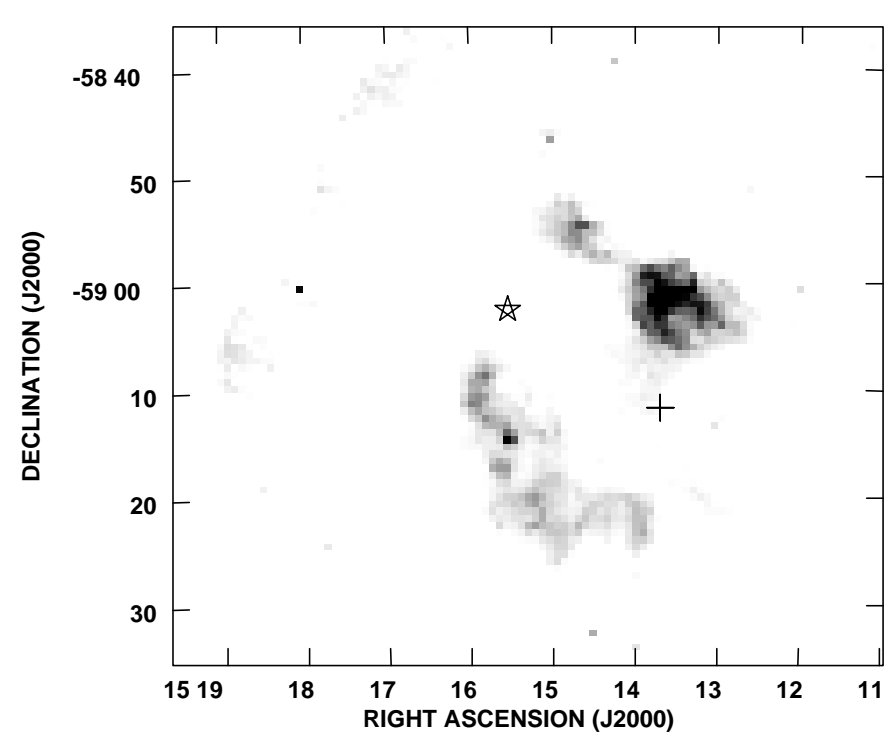

Fig. 1. Radiocontinuum emission at $1.4 \mathrm{GHz}$ as taken from Gaensler et al. (1999). The emission observed corresponds to the bilateral SNR G320.4-1.2. The angular resolution is $24^{\prime \prime} \times 21^{\prime \prime}$. The positions of WR 65 and WR 67 are indicated by a plus sign and a star respectively.

identification is tentative. No nebulosity related to WR 67 was detected (Marston 1997). The radio continuum images at 843 , 1420 and $5000 \mathrm{MHz}$ show no radio emission in the environs of both WR stars. The only extended radio source in the region is the bilateral SNR G320.4-1.2, located at a distance of about $5 \mathrm{kpc}$, including the bright nebula RCW 89 in the north half. Although the large ionizing flux of the WR stars should produce observable thermal radio continuum emission, in many cases it has not been detected (e.g. Cazzolato \& Pineault 2000 and references therein). Also non-thermal radio emission associated with WR stars has been detected in only few cases (Chapman et al. 1999).

Figure 1 (from Gaensler et al. 1999) shows the radiocontinuum emission at $1.4 \mathrm{GHz}$ in the region containing the stars under study.

\section{Observations and results}

The interferometric data were obtained with the ATCA during a session of $13 \mathrm{hs}$ on 1998 October 13, covering a field of about $2.4 \times 2.4$ through a 19 pointings mosaic. The pointings are chosen following an hexagonal grid, with a separation between grid points of about $16^{\prime}$ to satisfy the Nyquist sampling criterion. The hexagonal pointings configuration results in uniform sampling for the central $1.5 \times 1.5$ square. Thus our analysis is spatially restricted to the central area which is unaffected for borders effects.

To recover structures with the shortest spatial frequencies, the ATCA HI data were combined in the $u-v$ plane with the single dish (Parkes telescope) data from the Southern Galactic Plane Survey (SGPS; McClure-Griffiths et al. 2001). The data processing is described in Dubner et al. (2002). The HI cube cover LSR velocities between -150 and $+171 \mathrm{~km} \mathrm{~s}^{-1}$ at a velocity resolution of $1.1 \mathrm{~km} \mathrm{~s}^{-1}$ (channel separation of $\left.0.82 \mathrm{~km} \mathrm{~s}^{-1}\right)$, with an angular resolution of $4.0 \times 2$. 7 . The rms in the line-free channels is $\sim 30 \mathrm{mJy} /$ beam (approximately $0.5 \mathrm{~K}$ ).

We have examined in detail the HI $21-\mathrm{cm}$ line data in the whole observed velocity range looking for evidences of interaction between the WR stars and their local environs. Following previous experiences in similar studies (see for example Arnal et al. 1999) to distinguish the features possible associated with the stars from the background emission we required: that the structures remain detectable through a significant number of consecutive velocity channels; if a cavity is observed, then the WR stars must appear in projection inside the cavity (but not necessarily in the center); and finally, that the kinematical distance to the HI structure agrees, within the errors, with the photometric distance of the star.

In Dubner et al. (2002) it is presented the images of the $\mathrm{HI}$ distribution between $\simeq-80$ and $+92 \mathrm{~km} \mathrm{~s}^{-1}$ in channel maps traced every $5 \mathrm{~km} \mathrm{~s}^{-1}$. In this paper we confine our attention mostly to the LSR velocity range between $-35 \mathrm{~km} \mathrm{~s}^{-1}$ and $-60 \mathrm{~km} \mathrm{~s}^{-1}$, corresponding to the near kinematical distances of about 2.5 and $4 \mathrm{kpc}$, respectively, searching for evidences of bubbles or cavities close to the photometric distance of the WR stars. The presence of accelerated clouds at other velocities can be masked by structural components of the interstellar medium in this direction of the sky or by features associated with the SNR G320.4-1.2. Because of the distance ambiguity in the circular rotation galactic model, it is not possible to distinguish the features likely to be related to the WR stars at $\sim 3 \mathrm{kpc}$ from contribution of very distant galactic gas located near $\sim 9-10 \mathrm{kpc}$, that has the same LSR velocities. It is nevertheless expected that the contribution from distant gas is small based on the location of the galactic arms.

Figure 2 displays the HI emission distribution in the relevant velocity interval, between $-50 \mathrm{~km} \mathrm{~s}^{-1}$ and $-39 \mathrm{~km} \mathrm{~s}^{-1}$ (all velocities in this paper are referred to the LSR). Each image is the average of two consecutive spectral channels, spanning $1.6 \mathrm{~km} \mathrm{~s}^{-1}$. The velocity shown at the upper right corner of each image corresponds to the velocity of the first integrated channel. The stars WR 65 and WR 67 are indicated by a plus sign and a star, respectively. The upper and lower left corners have unreal border effects in the plotted contours, arising from the incomplete sampling towards the corners. The direction of the galactic plane is towards the NW corner.

From $\simeq-50 \mathrm{~km} \mathrm{~s}^{-1}$ to $\simeq-41 \mathrm{~km} \mathrm{~s}^{-1}$ it can be noticed the presence of a closed HI cavity centered near $15^{\mathrm{h}} 16^{\mathrm{m}} 30^{\mathrm{s}},-59^{\circ}$. This void is surrounded by an almost complete shell of enhanced HI emission. WR 67 appears projected against the HI void, practically located onto a small hole inside the larger cavity (see the images between $\simeq-41 \mathrm{~km} \mathrm{~s}^{-1}$ and $-44 \mathrm{~km} \mathrm{~s}^{-1}$ ), about $7^{\prime}$ west from the apparent geometrical center of the HI cavity.

There is a second, less conspicuous, HI minimum in the field: the elongated cavity centered near $15^{\mathrm{h}} 13^{\mathrm{m}},-59^{\circ}$ presents in the channel maps between $\sim-50$ and $-39 \mathrm{~km} \mathrm{~s}^{-1}$. This minimum appears connected with the main HI hole at velocities between $\sim-47$ and $-43 \mathrm{~km} \mathrm{~s}^{-1}$. WR 65 appears close but not exactly on the HI depression. It is projected onto a local maximum of the HI shell formed around the two minima. We have 


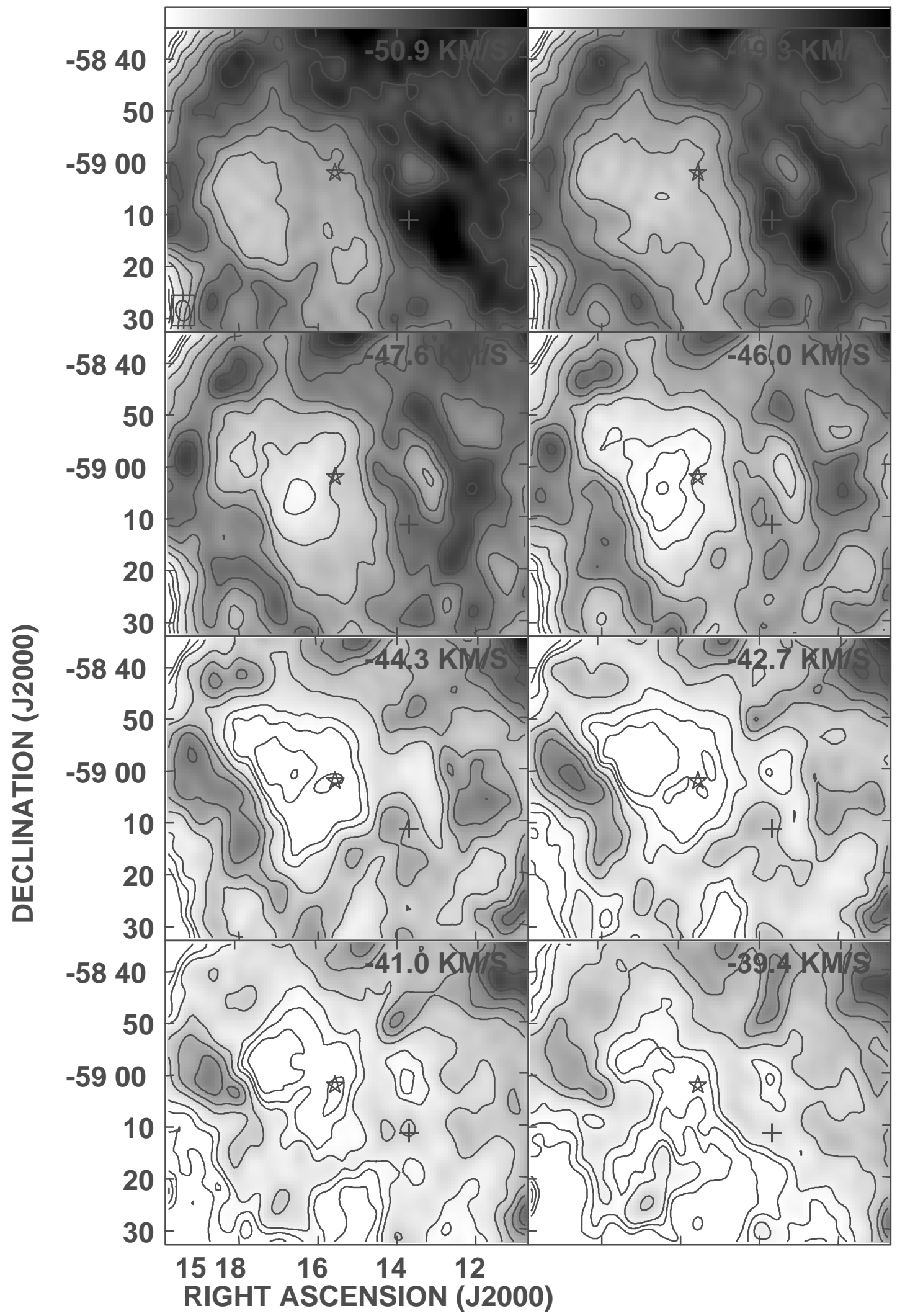

Fig. 2. Images of the $21 \mathrm{~cm} \mathrm{HI}$ emission between -50.9 and $-39.4 \mathrm{~km} \mathrm{~s}^{-1}$. Each map is the average of two consecutive channels $\left(1.6 \mathrm{~km} \mathrm{~s}^{-1}\right)$. The velocities shown in each map correspond to the velocity of the first integrated channel. They are: $-50.9,-49.3,-47.6,-46.0,-44.3$, $-42.7,-41.0$ and $-39.4 \mathrm{~km} \mathrm{~s}^{-1}$ from the upper left to the bottom right corner. The grayscale ranges from 1.8 to $6 \mathrm{Jy} / \mathrm{beam}$. The HI contours are 1.6, 1.8, 2, 2.5, 3, 3.5 4, and $4.5 \mathrm{Jy} /$ beam. The plus sign shows the position of WR 65 and the star identifies WR 67 . The angular resolution is $4^{\prime} \times 2$ '.7. 


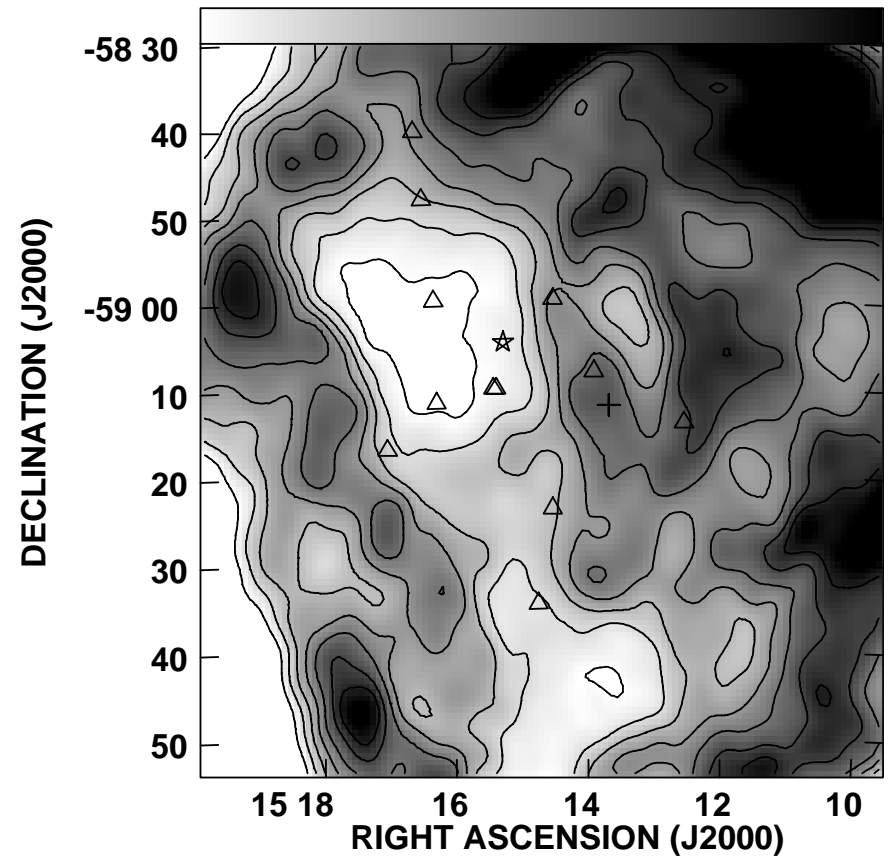

Fig. 3. The HI emission distribution averaged between -49 and $-39 \mathrm{~km} \mathrm{~s}^{-1}$. The grayscale goes from 2.0 to $4.2 \mathrm{Jy} / \mathrm{beam}$. The contours are 2, 2.3, 2.6, 2.9, 3.2, 3.6 and $3.9 \mathrm{Jy} /$ beam. The position of the open cluster Pis 20 (including WR 67) and WR 65 are indicated by a star and a plus sign respectively. The open triangles identify the OB stars belonging to the Circinus OB1 association. The beam is $4^{\prime} \times 2$ ' 7 .

searched in the whole data cube for other HI features with a morphology that may resemble a wind-blown bubble with the star WR 65 inside its boundaries, but the results were negative. The unfavorable location of WR 65 with respect to the cavities is not uncomon in the studies of the HI distribution around WR stars. There have been observed other cases where the WR star is overlapping a higher density edge of the associated bubble. See for example the case of WR 143, located close to the dense border of an HI cavity (Cazzolato \& Pineault 2000), or the cases of WR 5 and WR 123 (Arnal 1992) with a similar HI morphology. Besides, the fact that WR 65 is a member of Cir OB1 association may explain anomalies in the HI distribution and kinematics. As pointed out by Cazzolato \& Pineault (2000), OB associations can disturb the ISM by injecting energy into it, producing clouds motions and introducing velocity contamination in the HI data cube. In what follows, we will discuss both features taking into account the possibility that they are physically connected, although the association between the minor HI cavity and the WR 65 star is less convincing.

In Fig. 3 we display the average of twelve HI channels, between -49 and $-39 \mathrm{~km} \mathrm{~s}^{-1}$. A well defined HI shell encircling the large and the small cavities can be traced. This denser ring, with an average radius of $\simeq 25^{\prime}$ and a medium thickness of $\simeq 10^{\prime}$, opens to the $\mathrm{S}$. In this figure we have plotted in addition to WR 65, the open cluster Pis 20 (including WR 67) and other OB stars belonging to the Cir OB1 association. We want to investigate the impact on the surroundings of the stellar winds of all massive stars in the region (see Sect. 4).
Table 1. Observed and derived physical parameters.

\begin{tabular}{lc}
\hline \hline Velocity interval $\left(\mathrm{km} \mathrm{s}^{-1}\right)$ & -50 to -40 \\
Systemic velocity $\left(\mathrm{km} \mathrm{s}^{-1}\right)$ & $-45 \pm 2$ \\
Kinematical distance $(\mathrm{kpc})$ & $3 \pm 0.6$ \\
Expansion velocity $\left(\mathrm{km} \mathrm{s}^{-1}\right)$ & 7 \\
Average angular radius of the HI shell (arcmin) & 25 \\
Linear radius of the HI shell $(\mathrm{pc})$ & 22 \\
Missing HI mass $\left(M_{\odot}\right)$ & $\sim 1200$ \\
Shell HI mass $\left(M_{\odot}\right)$ & $\sim 1950$ \\
Swept-up HI mass $\left(M_{\odot}\right)$ & $\sim 1600$ \\
Dynamical age $(\mathrm{yr})$ & $1.7 \times 10^{6}$ \\
Kinetic energy $(\mathrm{ergs})$ & $8 \times 10^{47}$ \\
\hline
\end{tabular}

Table 1 summarizes the characteristic parameters of the HI feature. The velocity interval represents the kinematical velocity range where the gaseous structure appears better defined; the systemic velocity was chosen as the central one. For the LSR velocity of $(45 \pm 2) \mathrm{km} \mathrm{s}^{-1}$ we can estimate a kinematical distance (based on Fich et al.'s 1989 model) of (3.1 \pm 0.2$) \mathrm{kpc}$. The error in the distance estimate can be as big as $0.6 \mathrm{kpc}$ when non-circular motions of about $\pm 7 \mathrm{~km} \mathrm{~s}^{-1}$ are considered (Burton 1992). The expansion velocity listed is only a lower limit, since confusion from unrelated foreground and background emission impede the detection of possible "caps" to the expanding shell. The angular size of the HI shell was taken through the maxima of the shell and its linear dimension corresponds to the adopted distance for the HI feature. The HI mass deficiency was estimated for both cavities taken together and the HI mass in the shell was derived by integrating the column density distribution over the shell, within the velocity interval $(-50,-40) \mathrm{km} \mathrm{s}^{-1}$, assuming that the gas is optically thin. Because of the presence of neutral material that might be unrelated with the analized structures, the HI mass deficiency represents a lower limit, while the HI mass in the shell is an upper limit. The swept-up mass can be obtained as a mean value between these two masses estimates. Uncertainties of up to $40 \%$ in the estimated masses are caused by the error in distance, in the choice of the background level and in the integration boundaries. The kinetic energy was calculated from the sweptup mass and the estimated minimum expansion velocity.

\section{Infrared and molecular emission}

Figure 4 displays the comparison of the HI integrated between -49 and $-39 \mathrm{~km} \mathrm{~s}^{-1}$ (contours) with the IRAS highresolution (HIRES) image (Cao et al. 1997) at $100 \mu \mathrm{m}$ (greys). The HIRES image is the result of 20 iterations of the algorithm, smoothed to 2'.5. The plus signs indicate the IRAS point sources selected as protostellar candidates. The figure shows a good morphological agreement between the infrared emission and the HI feature. The emission at $60 \mu \mathrm{m}$ reproduces the main characteristics as that of $100 \mu \mathrm{m}$. The weaker emission 


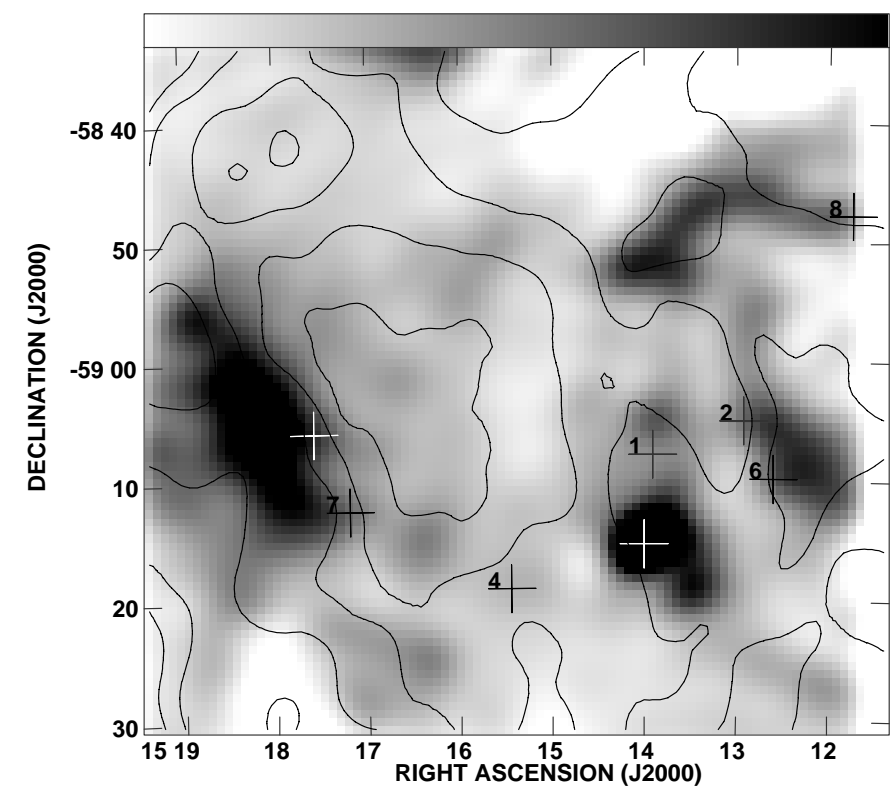

Fig. 4. Overlay of HIRES infrared image at $100 \mu \mathrm{m}$ with HI contours corresponding to emission integrated between -49 and $-39 \mathrm{~km} \mathrm{~s}^{-1}$, at levels of 2, 2.4, 3, and $3.6 \mathrm{Jy} /$ beam. The grayscale goes from 400 to $1000 \mathrm{MJy} / \mathrm{ster}$. The plus signs indicate the position of the IRAS point sources selected as protostellar objects candidates.

in both spectral bands are coincident and different portions of the HI shell have clear counterpart in the IR wavelenghts. It is remarkable how the eastern border of the larger cavity closely match the IR enhancements at $100 \mu \mathrm{m}$. The same is observed near the smaller cavity, towards the W. Note, for example, the very good IR-HI agreement observed in the elongated structure near $15^{\mathrm{h}} 13^{\mathrm{m}} 30^{\mathrm{s}},-58^{\circ} 50^{\prime}$, and to the $\mathrm{S}$, near $15^{\mathrm{h}} 13^{\mathrm{m}} 50^{\mathrm{s}}$, $-59^{\circ} 14^{\prime}$.

The dust temperature can be computed from the $100 \mu \mathrm{m} / 60 \mu \mathrm{m}$ color ratio using the relation

$\frac{S_{\lambda 1}}{S_{\lambda 2}}=\left(\frac{\lambda_{1}}{\lambda_{2}}\right)^{5} \frac{\mathrm{e}^{h c / \lambda_{2} k T_{\mathrm{c}}}-1}{\mathrm{e}^{h c / \lambda_{1} k T_{\mathrm{c}}}-1}$.

Where $\lambda_{1}$ and $\lambda_{2}$ correspond to $100 \mu \mathrm{m}$ and $60 \mu \mathrm{m}$ respectively. A dust emissivity exponent of 2 has been assumed.

From this relation a dust temperature of about $30 \mathrm{~K}$ is obtained. This is a typical value for dust heated by $\mathrm{OB}$ and WR stars (vanBuren \& McCray 1988) and is similar to the dust temperature derived for the shell associated with other WR stars (Arnal \& Mirabel 1991; Cichowolski et al. 2000; Arnal 2001).

This region, rich in massive stars, is ideal to look for a possible relation between stellar winds shocks and star formation. Thus, we have selected IRAS point sources with a spectrum compatible with protostellar candidates on the basis of the following selection criteria (that helps to discriminate against cold IRAS point sources, Junkes et al. 1992):

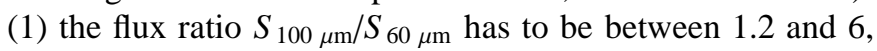
(2) $S_{25 \mu \mathrm{m}} \leq S_{60 \mu \mathrm{m}}$ and (3) the flux quality indices at $60 \mu \mathrm{m}$ and $100 \mu \mathrm{m}$ must fulfill: $Q_{60} \mu \mathrm{m}+Q_{100 \mu \mathrm{m}} \geq 4$. The positions of these infrared sources are indicated by a plus sign in Fig. 4
Table 2. IRAS point sources.

\begin{tabular}{llccc}
\hline \hline Object & $\begin{array}{l}\text { RA (J2000) } \\
\text { h m s }\end{array}$ & $\begin{array}{c}\text { Dec } \\
\circ^{\prime \prime \prime}\end{array}$ & $\begin{array}{c}\text { Flux [Jy] } \\
60 \mu \mathrm{m}\end{array}$ & $\begin{array}{c}\text { Flux [Jy] } \\
100 \mu \mathrm{m}\end{array}$ \\
\hline 1 & 151354.51 & -590739.0 & 36.95 & 138.10 \\
2 & 151255.23 & -590451.2 & 13.72 & 74.66 \\
3 & 15140.01 & -59158.7 & 227.20 & 547.10 \\
4 & 151526.73 & -591851.0 & 5.95 & 25.72 \\
5 & 151735.10 & -590553.0 & 17.49 & 72.66 \\
6 & 151235.89 & -590944.2 & 12.41 & 74.66 \\
7 & 151711.84 & -591222.3 & 15.37 & 54.65 \\
8 & 151144.84 & -584742.9 & 38.05 & 127.60 \\
\hline
\end{tabular}

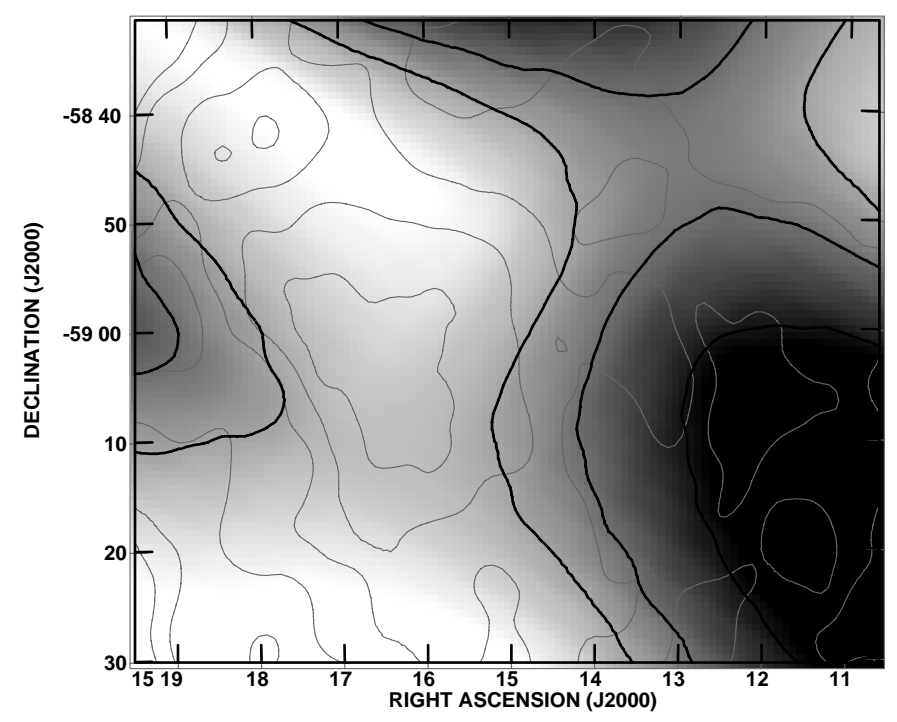

Fig. 5. Image of the $C O(J: 1-0)$ as taken from the Composite CO survey (Dame et al. 2001) averaged between -49 and $-39 \mathrm{~km} \mathrm{~s}^{-1}$. The grayscale range is $2.5-4.5 \mathrm{~K}$, and the contours (black lines) are 3, 3.6, and $4.6 \mathrm{~K}$. Some contours of the HI distribution averaged in the same velocity range have been included as gray lines.

and are listed in Table 2. The IR protostellar candidates appear strikingly distributed all around the dense border of the cavities.

Finally, in Fig. 5 we show a comparison between our $\mathrm{HI}$ distribution (gray contours) with the $\mathrm{CO}(\mathrm{J}: 1-0)$ data, as taken from the Composite CO survey (Dame et al. 2001) (angular resolution of $8^{\prime}$ ), integrated over the same velocity range as HI. Although the poorer angular resolution of the $\mathrm{CO}$ data precludes a detailed morphological comparison with the neutral gas, it can be observed that there is a good correlation between the two components of the ISM. This agreement provides additional support to the supposition that denser atomic and molecular gas has been encountered by the stellar winds to the $\mathrm{E}$ and to the $\mathrm{W}$, shaping the borders of the cavities. 


\section{Discussion}

In what follows we analyze the dynamical parameters to test the hypothesis that the combined action of WR 65 and WR 67 , and possibly other early-type stars of the stellar association, have created this two- cavities system, pilling up the material in the borders.

In order to do this, we first compare the mechanical energy liberated by the two WR stars into the ISM $\left(E_{\mathrm{w}}=\right.$ $\left.10^{43} \dot{M}\left(M_{\odot} / \mathrm{yr}\right) V_{\infty}^{2}\left(\mathrm{~km} \mathrm{~s}^{-1}\right) t_{\text {dyn }}(\mathrm{yr}) / 2\right)$ during the dynamical age of the bubble, with the kinetic energy of the swept-up gas $\left(E_{\mathrm{k}}=0.5 M_{\mathrm{s}} V_{\exp }^{2}\right)$. In these equations $M_{\mathrm{s}}$ is the swept-up mass, $\dot{M}$ is the mass-loss rate of the star, $V_{\infty}$ is the terminal velocity of its wind, $V_{\exp }$ is the expansion velocity of the shell and $t_{\text {dyn }}$ is the dynamical age of the structure. The dynamical age of an interstellar bubble can be estimated as $t=0.55 R / V_{\exp }$ (McCray 1983), where the constant represents a mean value between the energy and momentum conserving cases. $R$, the radius of the bubble in pc, was taken through the maxima of the envelope and $V_{\exp }$ is its expansion velocity in $\mathrm{km} \mathrm{s}^{-1}$. We obtain a dynamical age of $1.7 \times 10^{6} \mathrm{yr}$ for the bubble.

Using the values listed in Table 1 and the typical values of $\dot{M}$ and $V_{\infty}$ for both WR stars, i.e., $\dot{M} \sim 1.5 \times 10^{-5} M_{\odot} / \mathrm{yr}$ (Abbot et al. 1986) and $V_{\infty}=1200 \mathrm{~km} \mathrm{~s}^{-1}$ (van der Hucht 2001) for WR 65 , of type WC9, and $\dot{M} \sim 4.56 \times 10^{-5} M_{\odot} / \mathrm{yr}$ (Nugis \& Lamers 2000) and $V_{\infty}=1500 \mathrm{~km} \mathrm{~s}^{-1}$ (van der Hucht 2001) for WR 67, of type WN6, we obtain $E_{\mathrm{W}} \sim 2 \times 10^{51} \mathrm{ergs}$ and $E_{\mathrm{k}} \sim 8 \times 10^{47}$ ergs. We conclude that the mechanical energy provided by the WR stars are more than sufficient to create the observed HI feature.

The fact that the WR phase lasts about $5 \times 10^{5} \mathrm{yr}$, barely a $30 \%$ of the dynamical age of the bubble, indicates that it was created in previous evolutionary stages of the current WR star. We repeat the calculations with the typical parameters for a massive $\mathrm{O}$ type star progenitor $\left(\dot{M} \sim 2 \times 10^{-6} M_{\odot} / \mathrm{yr}, V_{\infty}=\right.$ $2000 \mathrm{~km} \mathrm{~s}^{-1}$, Garmany et al. 1981). The $E_{\mathrm{w}}$ turns out to be approximately $2.7 \times 10^{50}$ ergs showing that the progenitors of both WR stars would have been perfectly able to create the HI feature.

As a second step we evaluated the contribution of the young massive stars belonging to the open cluster Pis 20 . We retain only stars with spectral type earlier than type B2 because of their strong outflows of matter (Lozinskaya 1992). Based on the spectral types derived by Massey et al. (2001), the mean mass-loss rates from Garmany et al. (1981) and the terminal velocities estimated by Prinja et al. (1990), we obtain a total stellar energy input $E_{\mathrm{w}}$ of about $10^{48}$ ergs for all the early stars in the cluster, representing less than $0.1 \%$ of the mechanical energy injected by WR 65 and WR 67.

On the other hand, the properties of the stars members of the Cir OB1 association (plotted in Fig. 3) are less known, nevertheless using the distance modulus of $13 \mathrm{mag}$ for the Cir OB1 association (Lortet et al. 1987), the visual magnitudes of the OB stars, as derived by Orsatti \& Muzzio (1980), and assuming that the stars are in the main sequence, we can conjecture that the stars are on average of spectral type B7. Again, taking into account mean mass-loss rates and terminal velocities from Garmany et al. (1981) and Prinja et al. (1990) respectively, the total energy injected by all these stars to the ISM represents less than $0.1 \%$ of the mechanical energy input of the WR 65 and WR 67 stars.

Finally, we have performed a search in the literature for other early-types stars lieying within the limits of the HI cavities. The only one is HD135380 (B9VI) located at RA, $\operatorname{Dec}(\mathrm{J} 2000)=15^{\circ} 17^{\mathrm{m}} 28^{\mathrm{s}} .8,-59^{\circ} 03^{\prime} 11^{\prime \prime}$. Its contribution is minimum to the energy outflow in the region.

In summary, we suggest that the HI feature is a wind blown bubble almost exclusively created by the action of the stellar wind of WR 65, WR 67 and their O-type progenitors, in spite of the apparently inadequate location of WR 65 sitting on the denser border instead of within a hole. In addition, the multifrequency observations suggest that the action of the stellar winds shocks on the denser clouds encountered to the $\mathrm{E}$ and to the $\mathrm{W}$, may have triggered the formation of new stars. Detailed molecular observations are needed to locate the sites where the action of the stellar wind may have compressed surrounding cloudlets initiating the formation of the young stellar objects highlighted by the IR emission.

\section{Summary}

We have conducted a study of the distribution and kinematics of the neutral gas in the environs of the WR stars WR 65 and WR 67 with the Australia Telescope Compact Array. These data have revealed the existence of an interstellar bubble constituted by a double-cavity system surrounded by an almost complete shell. The major cavity has WR 67 located onto the deepest point in the hole. The second one, smaller and less conspicuous, is connected with the large cavity and has WR 65 projected onto the HI envelope. A shell of enhanced HI emission encircle both cavities. We suggest that the large HI bubble and the WR 67 star are physically related based on their excellent morphological and kinematical agreement. The case is less clear for WR 65 in view of the unfavorable location of the star.

Located at $3 \mathrm{kpc}$, this $\mathrm{HI}$ bubble has a diameter of $22 \mathrm{pc}$ and a swept-up mass of $1600 M_{\odot}$. Its observed expansion velocity of $7 \mathrm{~km} \mathrm{~s}^{-1}$ implies a kinematical age of $1.7 \times 10^{6} \mathrm{yr}$, larger than the duration of the WR phase. Therefore, not only the strong winds from the WR stars, but also the wind from their massive progenitors, must have been responsible for the formation of this bubble. Although we can not rule out the possiblity that other early- type stars in the neighbourhood have played some role in shaping the ISM in this region, their contribution through stellar winds is minimal compared to the powerful winds of the WR stars.

The IR emission has a good correspondence with the HI shell, mainly towards the E and W, where the IR emission perfectly matches the border of the HI cavity. The molecular counterpart also shows the same good correlation suggesting that preexisting neutral and molecular gas clouds have constrained the expansion and determined the shape of the HI bubble. The dust temperature of the HI shell, about $30 \mathrm{~K}$, is consistent with the value of dust heated by OB and WR stars. Several IRAS point sources selected with spectral characteristics of protostellar candidates were found projected on the periphery 
of the HI voids, suggesting a causal relationship between the stellar winds from massive stars and star formation.

Acknowledgements. We would like to thank B. Gaensler, A. Green and W. M. Goss for their contribution in the HI data acquisition and processing. We acknowledge the referee, Dr. Serge Pineault, for his suggestions that improved the presentation of this study. This research was funded through CONICET (Argentina) grant 4203/96 and grant UBACYT (Argentina) A013.

\section{References}

Abbott, D. C., Bieging, J. H., Churchwell, E., \& Torres, A. 1986, ApJ, 303, 239

Arnal, E. M. 1992, A\&A, 254, 305

Arnal, E. M. 2001, AJ, 121, 413

Arnal, E. M., \& Mirabel, I. F. 1991, A\&A, 250, 171

Arnal, M. E., Cappa, C. E., Rizzo, J. R., \& Cichowolski, S. 1999, AJ, 118,1798

Burton, W. B. 1992 in The Galactic Intertellar Medium, ed. D. Pfeniger, \& P. Bartholdi (Springer-Verlag), 1

Cao, Y., Terebey, S., Prince, T. A., \& Beichman, C. A. 1997, ApJS, 111,387

Cappa, C., Niemela, V., Herbstmeier, V., \& Koribalski, B. 1996, A\&A, 312,283

Cazzolato, F., \& Pineault, S. 2000, AJ, 120, 3192

Chapman, J., Leitherer, C., Koribalski, B., Bouter, R., \& Storey, M. 1999, ApJ, 518, 890

Cichowolski, S., Pineault, S., Arnal, E. M., et al. 2001, AJ, 122, 1938

Dame, T. M., Hartmann, D., \& Thaddeus, P. 2001, ApJ, 547, 792
Dubner, G. M., Gaensler, B. M., Giacani, E. B., Goss, W. M., \& Green, A. J. 2002, AJ, 123, 337

Fich, M., Blitz, L., \& Stark, A. A. 1989, ApJ, 342, 272

Gaensler, B. M., Brazier, K. T., Manchester, R., Johnston, S., \& Green, A. J. 1999, MNRAS, 305, 724

Garmany, C. D., Olson, G. L., Conti, P. S., \& vanSteenberg, M. E. 1981, ApJ, 250, 660

Gervais, S., \& St-Louis, N. 1999, AJ, 118, 2394

Junkes, N., Fürst, E., \& Reich, W. 1992, A\&A, 261, 289

Lortet, M.-C, Georgelin, Y. P., \& Georgelin, Y. M. 1987, A\&A, 180, 65

Lozinskaya, T. A. 1992, Supernovae and Stellar Wind in the Interstellar medium (New York: AIP), 279

Marston, A. P. 1997, ApJ, 475, 188

Marston, A. P., Chu, Y.-H., \& Garcia-Segura, G. 1994, ApJS, 93,229

Massey, P., DeGioa-Eastwood, K., \& Waterhouse, E. 2001, AJ, 121, 1050

McClure-Griffiths, N. M., Green, A. J., Dickey, J. M., et al. 2001, ApJ, 551,394

McCray, R. 1983, Highlights Astron., 5, 567

Nugis, T., \& Lamers, H. J. G. L. M. 2000, A\&A, 360, 227

Orsatti, A. M., \& Muzzio, J. C. 1980, AJ, 85, 265

Prinja, R. K., Barlow, M. J., \& Howarth, I. 1990, ApJ, 361, 607

Scaller, G., Schaerer, D., Meynet, G., \& Maeder, A. 1992, A\&AS, 96, 269

Turner, D. 1996, AJ, 111, 828

VanBuren, D., \& McCray, R. 1988, ApJ, 329, L93

van der Hucht, K. A. 2001, New Astron. Rev., 45, 135

Vázquez, R. A., Will, J.-M., Prado, P., \& Feinstein, A. 1995, A\&AS, 111,85 